\title{
NÍVEL DE ESTRESSE ENTRE PROFISSIONAIS DE ENFERMAGEM EM UM CENTRO CIRÚRGICO
}

\author{
Level of stress in nursing professionals of a surgical center
}

\author{
Nivel de estrés en los profesionales de enfermería en un centro quirúrgico
}

Tamires Leal da Silva' $\mathbb{C}^{\circ}$, Jacqueline Ramos de Andrade Antunes Gomes ${ }^{2}$ (C), Marcelo Moreira Corgozinho ${ }^{3 *}$

RESUMO: Objetivo: Avaliar o nível de estresse entre profissionais de enfermagem em centro cirúrgico. Método: Trata-se de estudo descritivo, exploratório, com abordagem quantitativa, que utilizou a Escala Bianchi de Stress para avaliar uma amostra de 50 participantes. Resultados: Houve predomínio do sexo feminino entre enfermeiros (100\%) e técnicos de enfermagem (90,7\%), da faixa etária entre 31 e 50 anos (76\%), do tempo de formação superior a 16 anos (64\%) e do tempo de atuação no centro cirúrgico acima de 16 anos (34\%). Os enfermeiros apresentaram escore médio de estresse em todos os domínios, com destaque para atividades relacionadas à administração de pessoal (escore 4,47), enquanto entre os técnicos de enfermagem predominou o baixo nível de estresse, com exceção do médio nível nas atividades de funcionamento adequado da unidade (escore 3,1). Conclusão: Os enfermeiros destacaram-se pelo médio nível de estresse, enquanto os técnicos pelo baixo nível de estresse. Destaca-se a necessidade de buscar estratégias de enfrentamento dos problemas de gestão administrativa, no caso dos enfermeiros, e de funcionamento do centro cirúrgico, para os técnicos de enfermagem. Palavras-chave: Equipe de enfermagem. Estresse psicológico. Centros cirúrgicos.

ABSTRACT: Objective: To assess the level of stress of nursing professionals in a surgical center. Method: This is a descriptive, exploratory study with a quantitative approach, based on the Bianchi Stress Scale to assess a sample of 50 participants. Results: Females were predominant among nurses (100\%) and nursing technicians (90.7\%), aged between 31 and 50 years (76\%), with more than 16 years of training (64\%) and working time in the operating room above 16 years (34\%). Nurses had an medium stress score in all domains, with emphasis on activities related to personnel administration (score 4.47), while among nursing technicians, a low level of stress was more common, except for the medium level in activities related to the functioning of the unit (score 3.1). Conclusion: Nurses had a medium level of stress, while technicians had a low level of stress. It is important to seek strategies for coping with administrative management problems in the case of nurses, and the operation of the surgical center for nursing technicians.

Keywords: Nursing, team. Stress, psychological. Surgicenters.

RESUMEN: Objetivo: Evaluar el nivel del estrese entre los profesionales de enfermería en centro quirúrgico. Método: Es un estudio descriptivo, exploratorio, con enfoque cuantitativo, que utilizó la Escala Bianchi de Stress para evaluar una muestra de 50 participantes. Resultados: Predominó el sexo femenino entre enfermeras (100\%) y técnicos de enfermería (90,7\%) con edades comprendidas entre 31 y 50 años ( $76 \%$ ), tiempo de formación mayor de 16 años ( $64 \%$ ) y tiempo en el centro quirúrgico mayor de 16 años (34\%). Las enfermeras habían presentado el puntaje medio del estrese en todos los dominios, con la prominencia para las actividades relacionadas con la administración de personal (puntaje 4.47), mientras que, entre los técnicos de enfermería, predominó el nivel bajo del estrese, con la excepción del nivel medio del estrese en las actividades del funcionamiento adecuado de la unidad (puntaje 3.1). Conclusión: Las enfermeras se destacaron por su nivel medio de estrés, mientras que los técnicos, por su bajo nivel de estrés. Se destaca la necesidad de buscar estrategias para la confrontación de los problemas de la gerencia administrativa, en el caso de las enfermeras, y del funcionamiento del centro quirúrgico, para los técnicos de enfermería. Palabras clave: Grupo de enfermería. Estrés psicológico. Centros quirúrgicos.

\footnotetext{
'Enfermeira especialista em centro cirúrgico pelo Programa de Residência Uniprofissional de Enfermagem em Centro Cirúrgico da Escola Superior em Ciências da Saúde (ESCS/Fepecs) - Brasília (DF), Brasil. 2Doutora em Ciências da Saúde pela Universidade de Brasília. Coordenadora do Programa de Residência Uniprofissional em Centro Cirúrgico da ESCS/Fepecs - Brasília (DF), Brasil.

${ }^{3}$ Doutor em Bioética pela Faculdade de Saúde da Universidade de Brasília. Preceptor e tutor da Residência Uniprofissonal de Enfermagem em Centro Cirúrgico da ESCS/Fepecs - Brasília (DF), Brasil.

*Autor correspondente: mmcorgozinho@gmail.com

Recebido: 13/08/2020 - Aprovado: 06/05/2021

https://doi.org/10.5327/Z1414-4425202100020002
} 


\section{INTRODUÇÃO}

Atualmente, no mundo do trabalho, dados os desafios do progresso industrial, a globalização, o desenvolvimento tecnológico e a comunicação virtual, os profissionais da saúde encontram-se expostos a situações que ultrapassam os limites das habilidades e capacidades desenvolvidas. Como resultado, o estresse pode ocasionar alterações no aspecto biopsicossocial do ser humano, prejudicando a saúde, a produtividade e o relacionamento com a família e o círculo social ${ }^{1}$.

O estresse pode ser definido como o desgaste geral do organismo causado pela pressão ou acúmulo de pressões psicofisiológicas, motivado por um estressor, que leva o indivíduo a um desequilíbrio na homeostase e o expõe a situações que o irritam, excitam ou amedrontam ${ }^{2-5}$.

A enfermagem é a profissão do cuidar, e os profissionais estão diretamente próximos dos pacientes e de seus familiares, convivendo diariamente com o sofrimento, a dor e a morte ${ }^{3}$. Além dos fatores mencionados, esses profissionais estão expostos, em seu ambiente de trabalho, a agentes químicos, físicos e biológicos, o que acarreta tensão, ansiedade e medo. O estresse ocupacional também está relacionado às altas demandas de trabalho, que resultam na redução da produtividade e da qualidade do trabalho, no aumento das taxas de absenteísmo e dos acidentes de trabalho e na maior rotatividade dos profissionais ${ }^{5-7}$.

Os profissionais de enfermagem que atuam no centro cirúrgico $(\mathrm{CC})$ possuem alta predisposição à incidência de estresse por desempenharem suas atividades em um ambiente fechado, com muitos riscos, diferentes rotinas, elevado nível de exigência técnica e alta produtividade. No período perioperatório, esses profissionais devem estar sempre atentos ao monitoramento do estado de saúde do paciente e preparados para atender às possíveis complicações. Além disso, fazem previsão e provisão de todos os recursos materiais a serem utilizados e dão suporte aos demais profissionais da equipe ${ }^{3}$.

Contudo, a relevância deste estudo decorre da necessidade de reconhecer os domínios de atuação profissional que geram maior carga de estresse na equipe de enfermagem que atua em CC, proporcionando a oportunidade de intervir com medidas de enfrentamento das situações estressoras ${ }^{8}$.

\section{OBJETIVOS}

- Avaliar o nível de estresse dos profissionais de enfermagem que atuam no CC de um hospital público do Distrito Federal (DF);
- Identificar as atividades mais desgastes entre os profissionais de enfermagem que atuam no CC de um hospital público do Distrito Federal (DF).

\section{MÉTODO}

Trata-se de estudo descritivo, de natureza exploratória, com abordagem quantitativa, realizado junto à equipe de enfermagem lotada no CC de um hospital público de Brasília (DF), que realiza cirurgias nas seguintes especialidades: oftalmologia, otorrinolaringologia, urologia, ginecologia, mastologia, plástica, vascular, ortopedia e urgência.

A amostra do estudo foi constituída de sete enfermeiros e 43 técnicos de enfermagem que atuam no CC do respectivo hospital nos períodos matutino, vespertino e noturno. Foram excluídos deste estudo os residentes de enfermagem e profissionais que se encontravam em férias e demais afastamentos legais.

Os dados foram coletados no mês de dezembro de 2019 e, como instrumento de coleta, utilizou-se a Escala Bianchi de Stress ${ }^{8}$, que tem a finalidade de medir o nível de estresse que o profissional atribui a cada atividade executada em seu ambiente de trabalho. É uma escala autoaplicável, validada e composta de duas partes:

- caracterização da amostra: sexo biológico, idade, cargo, unidade de trabalho, tempo de trabalho na unidade, turno de trabalho, tempo de formado, cursos de pós-graduação;

- estressores na atuação dos profissionais de enfermagem, com 51 itens em escala tipo Likert agrupados em seis domínios: domínio A - relacionamento com outras unidades e supervisores (nove situações); domínio B funcionamento adequado da unidade (seis situações); domínio C - administração de pessoal de enfermagem (seis situações); domínio D — assistência de enfermagem prestada ao paciente (quinze situações); domínio E - coordenação das atividades (oito situações); e domínio F — condições de trabalho (sete situações).

O instrumento foi testado quanto à confiabilidade interna por meio da aplicação do Alfa de Cronbach no Programa Statistical Package for the Social Sciences (SPSS) e obteve, na escala total, 0,96 (confiabilidade quase perfeita) e, nos domínios, acima de 0,70 (confiabilidade substancial), o que evidencia a consistência do instrumento de pesquisa. Como em outros estudos ${ }^{9,10}$, a escala foi aplicada para técnicos de enfermagem e enfermeiros por apresentar atividades exercidas por ambas as categorias e por haver a opção "não se aplica ou não faço — 0 ". 
No que tange à análise, os dados foram inseridos e computados no Microsoft Office Excel e, posteriormente, apresentados em tabelas para a análise descritiva das frequências absolutas. Realizou-se o escore para cada domínio nas duas categorias profissionais (enfermeiros e técnicos de enfermagem). Por meio da soma dos escores dos itens componentes de cada domínio e da divisão do resultado pelo número de itens, obtém-se o escore médio de cada domínio estudado. A variação dos escores de cada domínio é de 1 a 7 , sendo o nível de estresse padronizado com pontuação igual ou abaixo de 3 considerado como baixo nível de estresse), entre 3,1 e 5,9 como médio nível de estresse e igual ou acima de 6,0 como alto nível de estresse. O valor zero será reservado para os casos em que o profissional não executar a atividade abordada ou ela quando não se aplicar.
Este estudo foi apreciado e aprovado pelo Comitê de Ética em Pesquisa da Fundação de Ensino e Pesquisa em Ciências da Saúde (CEP/Fepecs), sob Parecer Consubstanciado n ${ }^{\circ}$ 3.685.587 e Certificado de Apresentação para Apreciação Ética (CAAE) $n^{\circ}$ 21152619.9.0000.5553, respeitando-se os termos da Resolução $n^{\circ}$ 466/2012 do Conselho Nacional de Saúde ${ }^{11}$.

\section{RESULTADOS}

Do total de participantes $(\mathrm{n}=50)$ que atuavam no CC, houve predomínio de profissionais do sexo biológico feminino tanto na categoria enfermeiro (100\%) quanto de técnico de enfermagem $(90,7 \%)$. Prevaleceu a faixa etária entre 31 e 50 anos

Tabela 1. Perfil dos profissionais de enfermagem do centro cirúrgico que compuseram a amostra.

\begin{tabular}{|c|c|c|c|}
\hline \multirow{2}{*}{ Variável } & \multicolumn{2}{|c|}{ Categoria Profissional } & \multirow{2}{*}{ Total $(\mathbf{n = 5 0})$} \\
\hline & Enfermeiro $(n=7)$ & Técnico de Enfermagem (n=43) & \\
\hline \multicolumn{4}{|l|}{ Idade (anos) } \\
\hline 20 a 30 & 0 & $2(4,65 \%)$ & $2(4 \%)$ \\
\hline 31 a 40 & $2(28,6 \%)$ & $13(30,23 \%)$ & $15(30 \%)$ \\
\hline 41 a 50 & $3(42,8 \%)$ & $20(46,51 \%)$ & $23(46 \%)$ \\
\hline Mais de 50 & $2(28,6 \%)$ & $8(18,6 \%)$ & $10(20 \%)$ \\
\hline \multicolumn{4}{|l|}{ Sexo biológico } \\
\hline Feminino & $7(100 \%)$ & $39(90,7 \%)$ & $46(92 \%)$ \\
\hline Masculino & 0 & $4(9,3 \%)$ & $4(8 \%)$ \\
\hline \multicolumn{4}{|c|}{ Tempo de formação (anos) } \\
\hline 2 a 5 & 0 & $3(6,97 \%)$ & $3(6 \%)$ \\
\hline 6 a 10 & $2(28,6 \%)$ & $6(13,95 \%)$ & $8(16 \%)$ \\
\hline 11 a 15 & 0 & $7(16,28 \%)$ & $7(14 \%)$ \\
\hline$>$ de 16 & $5(71,4 \%)$ & $27(62,8 \%)$ & $32(64 \%)$ \\
\hline \multicolumn{4}{|c|}{ Tempo na área (anos) } \\
\hline 2 a 5 & 0 & $2(4,65 \%)$ & $2(4 \%)$ \\
\hline 6 a 10 & $2(28,6 \%)$ & $10(23,25 \%)$ & $12(24 \%)$ \\
\hline 11 a 15 & 0 & $7(16,28 \%)$ & $7(14 \%)$ \\
\hline$>$ de 16 & $5(71,4 \%)$ & $23(53,5 \%)$ & $28(56 \%)$ \\
\hline Não respondeu & 0 & $1(2,32 \%)$ & $1(2 \%)$ \\
\hline \multicolumn{4}{|c|}{ Tempo de atuação atual (anos) } \\
\hline$<$ de 1 & $1(14,3 \%)$ & $9(20,93 \%)$ & $10(20 \%)$ \\
\hline 2 a 5 & $2(28,6 \%)$ & $7(16,28 \%)$ & $9(18 \%)$ \\
\hline 6 a 10 & 0 & $6(13,95 \%)$ & $6(12 \%)$ \\
\hline 11 a 15 & 0 & $7(16,28 \%)$ & $7(14 \%)$ \\
\hline$>$ de 16 & $4(57,1 \%)$ & $13(30,23 \%)$ & $17(34 \%)$ \\
\hline Não respondeu & 0 & $1(2,32 \%)$ & $1(2 \%)$ \\
\hline
\end{tabular}


(76\%), seguida daquela que engloba maiores de 50 anos (20,0\%), conforme apresentado na Tabela 1.

Quanto ao tempo de formação, a maioria tinha mais de 16 anos (64\%). Em relação ao tempo de atuação na área de CC, a maior parte tinha mais de 16 anos (56\%), seguida daqueles que tinham de 6 a 10 anos (24\%). Sobre o tempo de atuação no CC da instituição do estudo, 34\% tinham mais de 16 anos, seguidos daqueles com um ano (20\%).

$\mathrm{Na}$ Tabela 2, encontram-se os dados referentes ao nível de estresse detectado na equipe de enfermagem do CC, mensurado por meio da aplicação da Escala Bianchi de Stress. Na categoria dos enfermeiros, obtiveram-se escores entre 3,1 e 5,9, caracterizando nível médio de estresse em todos os domínios estudados na escala. O domínio de maior nível de estresse detectado foi "atividades relacionadas à administração de pessoal” (C. 4,47), seguido dos domínios "atividades relacionadas ao funcionamento adequado da unidade” (B. 4,14), "coordenação das atividades na unidade" (E. 3,76), "condições de trabalho” (F. 3,54), "relacionamento com outras unidades e supervisores" (A. 3,39) e "assistência de enfermagem prestada ao paciente" (D. 3,17).

Sobre o nível de estresse detectado na categoria profissional de técnicos de enfermagem, verificou-se predominância de baixo nível nos seguintes domínios: "relacionamento com outras unidades e supervisores" (A. 2,42), "assistência de enfermagem prestada ao paciente" (D 2,31), "condições de trabalho" (F. 2,27), "coordenação das atividades na unidade (E. $0,92)$ e "atividades relacionadas à administração pessoal" ( $C$ $0,48)$. Detectou-se médio nível de estresse entre os técnicos de enfermagem no domínio "atividades relacionadas ao funcionamento adequado da unidade" (B. 3,1).

Na Tabela 3 é possível identificar as atividades de maior carga de estresse contidas em cada domínio da escala de Bianchi. Os enfermeiros apresentaram médio nível de estresse em todos os domínios, com destaque para as "atividades relacionadas à administração pessoal" (C), tendo como principais atividades eleitas como maiores causadoras de estresse: "supervisionar as atividades da equipe" $(5,28)$ e "elaborar a escala mensal de funcionários" $(5,2)$. No domínio "controle de material usado" (B), os itens mais estressores foram: "controle de material usado" (4,57), "controle de equipamento" $(4,42)$ e "reposição de material" $(4,33)$.

Em relação aos técnicos de enfermagem, verificou-se que os itens geradores de maiores níveis de estresse no domínio "funcionamento adequado da unidade" (B) foram: "solicitação de revisão e consertos de equipamentos" $(5,44)$, "controle de equipamento" $(4,66)$ e "levantamento de quantidade de material existente na unidade" $(4,31)$.

Tabela 2. Nível de estresse entre profissionais de enfermagem do centro cirúrgico, segundo categoria profissional.

\begin{tabular}{|l|c|c|}
\hline \multirow{2}{*}{ Domínios } & \multicolumn{2}{|c|}{ Escala Bianchi de Stress* } \\
\hline Relacionamento com outras unidades e supervisores (A) & Enfermeiro & Técnico de enfermagem \\
\hline Atividades relacionadas ao funcionamento adequado da unidade (B) & 3,39 & 2,42 \\
\hline Atividades relacionadas à administração pessoal (C) & 4,14 & 3,1 \\
\hline Assistência de enfermagem prestada ao paciente (D) & 4,47 & 0,48 \\
\hline Coordenação das atividades na unidade (E) & 3,17 & 2,31 \\
\hline Condições de trabalho (F) & 3,76 & 0,92 \\
\hline
\end{tabular}

*Escala Bianchi de Stress: igual ou abaixo de 3,0 (baixo nível de estresse); entre 3,1 a 5,9 (médio nível de estresse) e igual ou acima de 6,0 (alto nível de estresse).

Tabela 3. Atividades mais estressoras nos domínios da Escala Bianchi de Stress, segundo categoria profissional.

\begin{tabular}{|c|c|c|c|}
\hline Categoria & Domínio & Situação & Média* \\
\hline \multirow{5}{*}{ Enfermeiro } & \multirow{2}{*}{$\begin{array}{c}\text { C - Administração de } \\
\text { pessoal }\end{array}$} & Supervisionar as atividades da equipe & 5,28 \\
\hline & & Elaborar escala mensal de funcionários & 5,20 \\
\hline & \multirow{3}{*}{$\begin{array}{l}\text { B - Funcionamento } \\
\text { adequado da unidade }\end{array}$} & Controle de material usado & 4,57 \\
\hline & & Controle de equipamento & 4,42 \\
\hline & & Reposição de material & 4,33 \\
\hline \multirow{3}{*}{$\begin{array}{l}\text { Técnico de } \\
\text { enfermagem }\end{array}$} & \multirow{3}{*}{$\begin{array}{l}\text { B - Funcionamento } \\
\text { adequado da unidade }\end{array}$} & Solicitação de revisão e consertos de equipamentos & 5,44 \\
\hline & & Controle de equipamento & 4,66 \\
\hline & & Levantamento da quantidade de material existente na unidade & 4,31 \\
\hline
\end{tabular}

*Escala Bianchi de Stress: igual ou abaixo de 3,0 (baixo nível de estresse); entre 3,1 a 5,9 (médio nível de estresse) e igual ou acima de 6,0 (alto nível de estresse). 


\section{DISCUSSÃO}

No presente estudo, os dados revelam o predomínio da participação do sexo biológico feminino na categoria de enfermagem, situação encontrada em outros estudos ${ }^{2,3,6,7,9,12-16}$. Esse fato condiz com o Conselho Federal de Enfermagem (Cofen), que, em $2015^{17}$, afirma que a equipe de enfermagem é predominantemente feminina $(84,6 \%)$ e ressalta o crescimento da população masculina nos últimos anos.

As profissionais de enfermagem exercem suas funções no trabalho além de seu papel de mãe e esposa no lar, ficando sobrecarregadas com o acúmulo de tarefas e atribuições que colabora para o surgimento de estresse ${ }^{16}$. Paralelamente a isso, a ética profissional sinaliza para uma conduta de enfermagem que desempenhe um trabalho afetivo em constante lapidação dos valores morais, promovendo empatia com a situação vivenciada pelo paciente ${ }^{18}$.

A faixa etária entre 41 e 50 anos prevaleceu entre as categorias da enfermagem. Estudos ${ }^{7,15}$ afirmam que profissionais com idade mais elevada possuem autoconfiança e maior segurança para desempenhar suas funções e enfrentar os agentes estressores do cotidiano laboral. Entretanto, uma pesquisa ${ }^{3}$ aponta o contrário, referindo que o envelhecimento, o desgaste fisiológico e o surgimento de doenças crônicas não transmissíveis estão associados à inadequação desses profissionais às rotinas do setor, tornando-os mais vulneráveis ao estresse.

Em relação ao tempo de formação e ao tempo de atuação na área, a maioria informou ter um período superior a 16 anos de experiência e mais de seis anos de atuação no CC da pesquisa. Portanto, trata-se de uma equipe bastante experiente na área de enfermagem em CC. Salienta-se que o tempo de atuação na área influencia no enfrentamento do estresse por contribuir para o aperfeiçoamento do profissional e prepará-lo para enfrentar situações que exijam conhecimento científico especializado, habilidade e segurança para desempenhar suas funções no ambiente de trabalho ${ }^{9,12}$.

Entre os enfermeiros, os domínios com maiores níveis de estresse foram o domínio $\mathrm{C}$, "atividades relacionadas à administração de pessoal", e o domínio B, "atividades relacionadas ao funcionamento adequado da unidade", o que vai ao encontro dos resultados verificados em estudos semelhantes ${ }^{9,12}$. Em relação ao domínio $C$, entre as atividades e os itens mais estressores, destacaram-se "supervisionar as atividades da equipe" e "elaborar escala mensal de funcionários". Esse domínio relaciona-se às atividades administrativas da enfermagem que exigem responsabilidade, empatia e dedicação para a realização de escala diária de trabalho, escala mensal de trabalho, escala de férias, mapa de cirurgias e gerenciamento dos recursos materiais e equipamentos do setor.
O relacionamento interpessoal é considerado um aspecto complexo, que colabora para o surgimento do estresse entre os profissionais ${ }^{3,9}$. Em um estudo ${ }^{19}$ realizado com profissionais enfermeiros que atuavam em CC, o índice de satisfação profissional foi baixo; a "interação profissional" foi o componente mais relevante e o "status profissional" o de menor relevância. Ressalta-se que, para minimizar os problemas inerentes à relação interpessoal, é necessário estimular o trabalho em equipe e afastar a individualização e a competitividade por meio de um convívio agradável, que contribua para prevenção do estresse e o aumento da qualidade de vida no trabalho?.

Os técnicos de enfermagem apresentaram médio nível de estresse apenas no domínio B, "funcionamento adequado da unidade”, e baixo nível de estresse nos demais. No entanto, outro estudo ${ }^{3}$ similar não evidenciou estresse na maioria dos profissionais de enfermagem, apesar de o CC ser um setor que contribui para seu surgimento.

Quanto às atividades relacionadas ao "funcionamento adequado da unidade" (domínio B), verificou-se médio nível de estresse para ambas as categorias da enfermagem, sendo as situações mais estressantes descritas: "controle de material usado", "controle de equipamento", "reposição de material", "solicitação de revisão e consertos de equipamentos" e "levantamento da quantidade de material existente". A escassez de materiais e o funcionamento inadequado dos equipamentos proporcionam desgaste físico e psicológico aos profissionais da equipe de enfermagem e, consequentemente, ocasionam estresse por eles se preocuparem com a qualidade da assistência prestada ao paciente cirúrgico ${ }^{15}$.

A exposição dos profissionais a situações potencialmente estressoras pode desencadear estados de estresse, com interferência no desempenho das atividades e na qualidade da assistência prestada ${ }^{2,15}$. É necessário que os profissionais de enfermagem trabalhem alinhados e em equipe a fim de garantir o adequado funcionamento da unidade, a segurança do paciente e a menor exposição aos fatores estressores ${ }^{9}$.

Considera-se como limitação do estudo a ausência de comparação dos níveis de estresse entre CC de diferentes perfis de atendimento. Contudo, destaca-se que mesmo com a presença de fatores estressores a equipe de enfermagem, na sua função tanto gerencial quanto assistencial, contribui decisivamente para práticas assistenciais seguras em CC.

\section{CONCLUSÕES}

A análise dos resultados permitiu concluir que os enfermeiros participantes do estudo apresentaram nível médio de estresse 
em todos os domínios estudados, enquanto entre os técnicos de enfermagem predominou nível baixo de estresse, com exceção do nível médio de estresse no domínio "funcionamento adequado da unidade". O domínio com maior estresse entre os enfermeiros está relacionado à administração de pessoal e ao funcionamento adequado da unidade, como, por exemplo, as atividades de gerenciamento da equipe, bem como as burocráticas relativas ao ambiente cirúrgico. Em relação ao funcionamento da unidade, as atividades de previsão, provisão e controle de materiais e equipamentos são as mais desgastantes e causadoras de estresse, provavelmente pelo fato de os profissionais conviverem rotineiramente com a escassez de materiais e a baixa qualidade dos equipamentos.

Espera-se que os resultados apresentados neste estudo possam conscientizar os profissionais e a equipe gestora sobre os fatores causadores de estresse e subsidiar estratégias de enfrentamento das situações geradoras de estresse entre os profissionais de enfermagem que atuam em CC.

\section{REFERÊNCIAS}

1. Organização Pan-Americana da Saúde (OPAS). Organização Mundial da Saúde (OMS). Estresse no ambiente de trabalho cobra preço alto de indivíduos, empregadores e sociedade [Internet]. Brasília: Organização Pan-Americana da Saúde; 2016 [acessado em 28 jul. 2019]. Disponível em: https://www.paho.org/bra/index.php?option=com_content\&vie $\mathrm{w}=$ article\&id=5087:estresse-no-ambiente-de-trabalho-cobra-precoalto-de-individuos-empregadores-e-sociedade\&ltemid=839

2. Sampaio LR, Oliveira LC, Pires MFDN. Empatia, depressão, ansiedade e estresse em profissionais de saúde brasileiros. Ciênc Psicol. 2020;14(2):e-2215. https://doi.org/10.22235/cp.v14i2.2215

3. Chiavone FBT, Gomes ATL, Rodrigues CCFM, Ferreira LL, Salvador PTCO, Santos VEP. Stress levels of the surgical center nursing team: a cross - sectional study. Online Braz J Nurs. 2018;17(1):87-96. https://doi.org/10.17665/1676-4285.20185902

4. Prado CEP. Estresse ocupacional: causas e consequências. Rev Bras Med Trab. 2016;14(3):285-9. https://doi.org/10.5327/Z1679-443520163515

5. Souza RC, Silva SM, Costa MLAS. Estresse ocupacional no ambiente hospitalar: revisão das estratégias de enfrentamento dos trabalhadores de enfermagem. Rev Bras Med. 2018;16(4):493-502. https://doi. org/10.5327/Z1679443520180279

6. Soares LMP, Oliveira VC, Sousa LAA. Qualidade de vida dos profissionais atuantes no centro cirúrgico. Rev Psicol Saúde Debate. 2017;3(2):15970. https://doi.org/10.22289/V3N2A12

7. Souza ISN, Silva FJ, Gomes RLV, Frazão IS. Situações estressantes de trabalho dos enfermeiros de um hospital escola. Rev Enferm UFSM. 2013;3(2):287-95. https://doi.org/10.5902/217976928322

8. Bianchi ERF. Escala Bianchi de Stress. Rev Esc Enferm USP. 2009;3(Esp.):1055-62. https://doi.org/10.1590/S0080-62342009000500009

9. MirandaSMM. Onível de estresse do profissional de enfermagem que atua no centro cirúrgico em um hospital privado do Distrito Federal [trabalho de conclusão de curso]. Brasília: Centro Universitário de Brasília, Curso de Enfermagem, Faculdade de Ciências da Educação e Saúde (FACES); 2017.

10. Rosso E, Loures Junior EJ, Aggio CM, Trincaus MR, Possolli GT, ZanotiJeronymo. Avaliação do nível de estresse entre os profissionais de enfermagem atuantes no SAMU de Guarapuava - PR. BJSCR [Internet]. 2014 [acessado em 20 jun. 2020];7(1):13-7. Disponível em: https:// www.mastereditora.com.br/periodico/20140602_103709.pdf
11. Brasil. Conselho Nacional de Saúde. Resolução n 466, de 12 de dezembro de 2012. Diretrizes e normas regulamentadoras de pesquisas envolvendo seres humanos. Diário Oficial da União. 2013.

12. Kirhhof RS, Oshôa LM, Bublitz S, Lopes LFD, Squiavenato MCA. Nível de estresse entre enfermeiros de um hospital filantrópico de médio porte. Rev Enferm UFSM. 2016;6(1):29-39. https://doi. org/10.5902/2179769217829

13. Monte PF, Lima FET, Neves FMO, Studart RMB, Dantas RT. Estresse dos profissionais enfermeiros que atuam na unidade de terapia intensiva. Acta Paul Enferm. 2013;26(5):421-7. https://doi.org/10.1590/ S0103-21002013000500004

14. Soratto MT, Souza MP, Mattos SB, Ceretta LB, Gomes KM, Correa SM. 0 estresse da equipe de enfermagem no centro cirúrgico. RIES [Internet]. 2016 [acessado em 28 fev. 2020];5(1):179-92. Disponível em: http://periodicos.uniarp.edu.br/index.php/ries/ article/view/717/440

15. Oliveira EB, Gallasch CH, Silva Junior PPA, Oliveira AVR, Valério RL, Dias LBS. Estresse ocupacional e burnout em enfermeiros de um serviço de emergência: a organização do trabalho. Rev Enferm UERJ. 2017;25:e28842. https://doi.org/10.12957/reuerj.2017.28842

16. Pinto APCM, Silva MF, Azevedo ACB, Rodrigues CCFM, Salvador PTCO, Santos VEP. Estresse no cotidiano dos profissionais de enfermagem: reflexos da rotina laboral hospitalar. Rev Enferm UFSM [Internet]. 2016 [acessado em 26 fev. 2020];6(4):548-58. Disponível em: https://periodicos.ufsm.br/reufsm/article/view/21779. https:// doi.org/10.5902/2179769221779

17. Conselho Federal de Enfermagem (COFEn). 0 perfil da enfermagem no Brasil. Brasília: COFEn; 2015.

18. Grison P, Aguiar D, Moser G, Hanauer M, Klein S. Disposição afetiva para o cuidado na recuperação: o cotidiano da equipe de enfermagem. Rev SOBECC [Internet]. 2020 [acessado em 8 nov. 2020];25(3):15970. Disponível em: https://revista.sobecc.org.br/sobecc/article/ view/595. https://doi.org/10.5327/Z1414-4425202000030006

19. Gouveia L, Ribeiro V, Carvalho R. Satisfação profissional de enfermeiros que atuam no bloco cirúrgico de um hospital de excelência. Rev SOBECC [Internet]. 2020 [acessado em 8 nov. 2020];25(1):33-41. Disponivel em: https://revista.sobecc.org.br/sobecc/article/view/574. https://doi.org/10.5327/Z1414-4425202000010006 